\title{
Waxy and Soluble Starch Synthasell-3 Alleles Regulating Rice Resistant Starch Contents from Different Processing Status
}

hui you

Southwest University of Science and Technology https://orcid.org/0000-0001-9129-0098

Liang Xu

Southwest University of Science and Technology

Ouling Zhang

Southwest University of Science and Technology

Xunchao Xiang ( $\nabla$ xiangxunchao@swust.edu.cn )

https://orcid.org/0000-0002-5926-3011

Original article

Keywords: Rice (Oryza sativa L.), Resistant Starch, Wx, SSII-3, Physicochemical properties

Posted Date: February 12th, 2020

DOI: https://doi.org/10.21203/rs.2.23341/v1

License: (c) (1) This work is licensed under a Creative Commons Attribution 4.0 International License. Read Full License 


\section{Abstract}

\section{Background}

Resistant Starch (RS) is a healthy dietary fiber that has functions of regulating diabetes, hypertension and obesity. Previous studies mainly focused on investigating RS in raw rice or cooked rice separately, which may receive different results.

\section{Results}

In this study, ninety-nine lines from a recombinant inbred line (RIL) were selected to investigate the effects of starch synthesis-related genes on the RS content in different process status. RS content in rice will change by different processing ways. Waxy $(W x)$ played an important role in controlling RS content and $W x^{a}$ could elevate RS content, and soluble starch synthasell-3 (SSII-3) had an impact on RS2. Additionally, interaction of $W x$ and SSII-3 was responsible for variations of RS content in three sample types and RS2. $W x$ could affect RS in cooked rice and retrograded rice under the same SSII-3 allele. Moreover, the correlation analysis results indicated that RS was closely relative with many indexes of physicochemical properties.

\section{Conclusions}

Wx and SSII-3 could regulate RS content of rice, but SSII-3 especially affected RS2. The findings herein should provide useful information for molecular breeding of rice RS.

\section{Background}

Resistant starch (RS) is a sum of starch or starchy food products that cannot be digested in the small intestine of healthy individuals but fermented in the large intestine by microbial flora (Asp 1992). According to the botanical origin and nature, RS can be classified further into five subtypes: RS1, RS2, RS3, RS4 and RS5 (Xia et al. 2018). RS2 and RS3 are the main types found in raw and cooked rice, respectively. RS2 is made up of native starch granules whose formation and structure protects it from digestion and is usually present in raw potato, green banana and uncooked starch (Hernández et al. 2008). The physiological functions and commercial values of RS2 are few because of its rapid digestion after being heating processed and cooked. RS3 consists of retrograded starch, mainly the recrystallized amylose, formed during cooling of gelatinized starch and cooked foods that are stored at room or low temperature (Sharma et al. 2008), which is widely used in food processing industry.

With the development of living standards and changes of life style, the number of people with type-2 diabetes are increasing. Consumption of food with high RS can help to prevent from diabetes, because of its slow digestion and absorption by the small intestine decreases postprandial glucose and insulin responses (Raigond et al. 2015). Meanwhile, RS can be decomposed into short chain fatty acids (SCFA) such as acetic acid, propionic acid and butyric acid in the large intestine, which can improve the health of 
large intestine and prevent the occurrence of intestinal inflammatory diseases and colon cancer (Robertson et al. 2000; Topping et al. 2008). Consumption of RS can increase satiety and reduce calorie intake, which helps weight management (Raigond et al. 2015). Therefore, it is important to elevate the content of RS in food.

Rice (Oryza sativa L.) feeds more than half of the world's population as staple food and is the main source of nutrition and carbohydrates for many people. RS content is generally under $3 \%$ in hot cooked rice cultivars, which is not enough to confer the associated health benefits (Frei et al. 2003; Hu et al. 2004). Therefore, many scholars have focused on the promotion of RS content in rice varieties. In the past decade, many mutants or varieties with elevated RS have been identified, such as RS111 (Yang et al. 2006), Gaomi 2 (Kim et al. 2006), Jiangtangdao 1 (Yang et al. 2012), b10 (Zhou et al. 2016).

Starch biosynthesis is a sophisticated process involving multiple enzymes, including ADP-glucose pyrophosphorylase (AGPase), granule-bound starch synthase (GBSSI), soluble starch synthase (SS), starch branching enzymes (SBEs), isoamylase (ISA) and pullulanase (PUL) (Tian et al. 2009). Among them, GBSSI is encoded by the Waxy (Wx), which plays an important role in determining the synthesis of amylose. SS, SBEs, ISA and PUL can control the synthesis of amylopectin. RS content can be affected by the ratio of amylose to amylopectin and the fine structure of starch (Eggum et al. 1993; Hu et al. 2004). Thus, these starch synthesis-related genes (SSRGs) naturally have great influence on the synthesis of RS. A high-RS mutant has been developed by influencing the expression of starch branching enzyme 3 (SBE3) (Yang et al. 2012). Zhou et al. (2016) believed that soluble starch synthaselll-2 (SSIII-2) can regulate RS content and further revealed that RS yield is dependent on the high expression of the $\mathrm{Wx}^{\mathrm{a}}$ allele, which is prevalent in indica varieties. In other cereals, the down- regulation of soluble starch synthasell-3 (SSII-3) and SBE could increase RS content in barley (Topping et al. 2003; Bird et al. 2004) and wheat (Hazard et al. 2014; Ahmed et al. 2015). Due to the molecular basis underlying the RS synthesis is largely unclear, identifying the RS genes is crucial for clarifying the RS synthesis and for the breeding of high-RS varieties.

It is generally considered that eating and cooking qualities (ECQs) are the most crucial rice qualities, which are mainly comprised of three physicochemical properties: apparent amylose content (AAC), gel consistency (GC), and gelatinization temperature (GT) (Tian et al. 2009). In recent years, because of its advantages of simple and rapid, the rapid visco analyzer (RVA) has been widely employed to evaluate the rice ECQs. The AAC shows a significantly positive correlation with RS content (Kong et al. 2015). Most of rice varieties with eminent RS content tend to have high AAC and poor taste, which is not acceptable to most consumers. Therefore, it is an important goal to study the relationships between RS and physicochemical properties in rice, which can make a foundation for obtaining rice varieties with elevated RS content and good taste. In this study, the recombinant inbred lines (RILs) were employed to study the effects of Wx and SSII-3 on RS content, and the relationships between RS and physicochemical properties were analyzed, aiming to provide useful information for molecular breeding of rice RS.

\section{Materials And Methods}




\section{Plant Materials}

In present study, CG133R (Oryza sativa ssp. indica, a restorer line) and Javanica22 (a natural mutant from javanica rice variety Xiangdali), whose SSRGs differed in ADPglucose pyrophorylase small unit (AGPsma), ADPglucose pyrophorylase large unit (AGPlar), Wx, SSII-3, soluble starch synthaselll-1(SSIII-1) and soluble starch synthaselV-2 (SSIV-2) (Fig. 1), were used to construct RIL. At seventh generation, a total of 99 lines with homozygous genetic background of SSRGs were identified from 142 lines and then a single plant was harvested from each line and taken as experimental materials when they were mature. All the materials were grown in an experimental agricultural field of the Southwest University of Science and Technology (Sichuan, China) and maintained normal management.

\section{Methods}

\section{Sample preparation}

Harvested rice grains were air-dried and stored at room temperature for 3 months to balance water before analyses. Rice was dehulled by using a Dehuller (TR-200, kett, Japan). The brown rice was polished by using polisher (kett, Japan) and subsequently ground to flour in a Laboratory Mill 300 (Perten, Sweden) with a 100 mesh sieve for measuring RS content and physicochemical properties.

\section{Measurement Of Resistant Starch And Physicochemical Properties}

Cooked rice was prepared according to the Zhou's method (Zhou et al. 2016) and taken a part of cooked rice stored at $4{ }^{\circ} \mathrm{C}$ for a week to product retrograded rice. In this work, RS content was measured in three sample types, including milled samples (raw rice), cooked rice and retrograded rice by using RS Rapid Assay Kit (Megazyme, International Ireland Ltd.), according to the manufacture's assay procedure. Each starch sample was measured in triplicate.

AAC and GC were measured according to the standard of Chinese Ministry of Agriculture, NY/T 2639 2014, and Chinese national standards, GB/T 17891 - 1999, respectively. GT was estimated by differential scanning calorimetry (DSC) according to methods reported previously (Yan et al. 2010). In order to measure sample percentage of retrogradation ( $R \%$, the gelatinized starch was stored at $4^{\circ} \mathrm{C}$ for 7 days. Afterwards, it was equilibrated to room temperature for $1 \mathrm{~h}$ and then with the same thermal program as the measurement of GT. R was calculated by the following formula:

$\mathrm{R} \%=\Delta \mathrm{Hr}$ (enthalpy of retrogradation) $/ \Delta \mathrm{Hg}$ (enthalpy of gelatinization)

Each index of per sample was measured in duplicate. 


\section{Measurement of RVA profile characteristics}

A RVA (Model No. RVA4500, NewPortSci. Co. Warriewood, Australia) was used to estimate RVA profile characteristics in accordance with the American Association of Cereal Chemists (AACC) operational procedure. Three original parameters obtained from the RVA profile as following: peak viscosity (PKV), hot paste viscosity (HPV), cool paste viscosity (CPV); and three secondary parameters including: breakdown viscosity (BDV=PKV-HPV), setback viscosity ( $\mathrm{SBV}=\mathrm{CPV}-\mathrm{PKV})$, consistence viscosity $(\mathrm{CSV}=\mathrm{CPV}-\mathrm{HPV})$. Besides that, the pasting temperature $(\mathrm{PaT})$ and pasting time (PeT) also were recorded. Each starch sample was measured in duplicate.

\section{DNA Extraction And Genotyping}

About $100 \mathrm{mg}$ fresh young leaf was sampled from each plant and grounded using a Fastprep Sample Rapid Crushing System (MP Biomedicals, Santa Ana, CA, USA). The genome DNA was extracted with the CTAB method (Murray and Thompson, 1980).

A total of $10 \mu \mathrm{L}$ reaction mixtures using the Golden Easy PCR System (TIANGEN, Beijing, China), were employed to identify the genotypes of 18 SSRGs alleles between two parents by using the primers designed by Tian et al. (2010); Cai et al. (2002) and Zhou et al. (2016) (Additional file 2: Table S1). And then, the molecular markers of differential genes between two parents were selected to detect the genotypes of 99 lines. All the primers were synthesized by Sangon Biotech (Shanghai, China). Polymerase chain reactions (PCR) ran on an Eppendorf Thermal Cycler (Mastercycler ${ }^{\circledR}$ nexus GSX1, Germany). $5 \mu \mathrm{L}$ of each amplification products of CAPS marker was digested with corresponding restriction endonuclease in a total of $15 \mu \mathrm{L}$ enzymatic system including $1.5 \mu \mathrm{L} 10 \times$ buffer, $8 \mu \mathrm{L} \mathrm{ddH_{2 } \mathrm { O }}$ and $0.5 \mu \mathrm{L}$ restriction endonuclease. The digestion reactions were performed by using an Eppendorf Thermal Cycler at $37^{\circ} \mathrm{C}$ for $3-4 \mathrm{~h}$. All the amplified products were detected on a $3 \%$ agarose gel in $0.5 \times$ Tris-Borate EDTA (TBE) buffer using GreenView (Applied BioProbes, Rockville, MD, USA).

\section{Statistical Analyses}

Analysis of variance (ANOVA) and multiple comparisons of the Duncan method were performed with the Statistical Product and Service Solutions software version 22 (SPSS, https://www.ibm.com/analytics/cn/zh/technology/spss/) after the date being classified into groups according to the genotyping results. In addition, the correlation analysis between RS content and physicochemical properties was conducted by using SPSS 22 .

\section{Results}




\section{Analysis on genetic background of materials and grouping of RIL}

Polymorphism of eighteen SSRGs had been detected between two parents and they differed in 6 genes (AGPsma, AGPlar, SSIII-1, SSIV-2, Wx and SSII-3). Genetic analysis on SSRGs of the 99 lines came from the RIL showed that there were different allelic genotypes for the 6 genes (Fig. 1). Among the experimental population, statistically significant differences on RS were only detected in different $W x$ alleles and SSII-3 alleles $(P<0.05)$ (Additional file 2: Table S2). So we mainly focused on the effects of $W x$ and SSII-3 alleles for RS content. Diverse groups were obtained according to different alleles among 99 lines, and were designated as follows: to $W x$ alleles: type $C$ (came from CG133R), type J (came from Javanica 22), respectively; to SSII-3 locus: type I (same as Javanica 22) and type II (same as CG133R), respectively (Fig. 1).

\section{Variation of RS content of rice RIL and their parents}

A t-test was implemented in order to compare the difference of parents on RS content. The result showed that the parents differed significantly from each other on the RS content in milled samples (RSm), the RS content in cooked rice (RSc) and the RS content in retrograded rice (RSr) at 0.05 level (Table 1). RS content in three sample types showed a wide variations among the RIL, especially RSm, with an average of $10.61 \%$ and ranging from $0.03 \%$ to $29.42 \%$ (Table 1 ). Compared with the RSm, the average of RSc and RSr decreased by $9.88 \%$ and $9.55 \%$, respectively. The difference of RS content between milled samples and cooked rice was significant in RIL $(P<0.01)$, but there was no statistical difference on RS content between cooked rice and retrograded rice at 0.05 level (Additional file 1: Figure S1). This fact indicated that RS contents of the different lines were decreased sharply after cooking, but no distinct variation was observed in RS content after retrogradated process. Therefore, the subtype of RS in the RIL was mainly RS2, and less RS3.

Table 1

Distribution of three types of RS in RIL and their Parents.

\begin{tabular}{|lllll|}
\hline Types & RIL & & Javanica 22 & CG133R \\
\cline { 2 - 5 } & Rang & Mean \pm SD & Mean \pm SD & Mean \pm SD \\
\hline RSm(\%) & $0.03-29.42$ & $10.61 \pm 5.48$ & $2.12 \pm 0.05^{*}$ & $5.32 \pm 0.15$ \\
\hline RSc(\%) & $0.00-1.71$ & $0.73 \pm 0.39$ & $0.01 \pm 0.01^{*}$ & $1.21 \pm 0.04$ \\
\hline RSr(\%) & $0.00-2.21$ & $1.06 \pm 0.50$ & $0.2 \pm 0.00^{*}$ & $1.96 \pm 0.13$ \\
\hline $\begin{array}{l}\text { Note: } \text { RSm, resistant starch content in milled sample; RSc, resistant starch content in cooked rice; } \text { RSc, } \\
\text { resistant starch content in retrograded rice. * indicates P< } 0.05 \text {, according to Student's t-test. }\end{array}$ \\
\hline
\end{tabular}




\section{Effects of $W x$ on RS in the RIL}

Wx genotype of parents were identified by utilizing molecular marker PCR-AcCl and $W x \mathrm{M} 1$ and result indicated the CG133R and Javanica22 with the background of $W x^{a}$ and $w x$, respectively (Fig. 1, Additional file 1: Figure S2). Among the 99 experimental lines, $W x$ alleles were divided two groups: type $C$ and type $J$. Effects of $W x$ on rice RS were investigated by ANOVA. The result demonstrated that there were significant differences on RSm, RSc and RSr under different $W x$ alleles at 0.01 level (Fig. 2a). The average of RSm, RSc and RSr of type $C$ increased by $9.54 \%, 0.85 \%$ and $1.21 \%$, respectively, compared with that of type J. The result suggested that $W x^{a}$ could improve RS content in three sample types.

Furthermore, the differences between RSm and RSc, RSr and RSc were calculated. RSa= RSm - RSc, RSb= $\mathrm{RSr}$ - RSc. RSa mainly was RS2 and RSb mainly was RS3. Result of ANOVA revealed that RSa varied significantly under different alleles of $W x$, as did RSb ( $P<0.01)$ (Fig. 2b). RSa and RSb of type J $(2.53 \%$, $0.05 \%$, respectively) also were extremely significantly lower than that of type $C(11.21 \%, 0.41 \%)$ at 0.01 level. This result indicated that $W x$ could affect RS 2 and RS 3 and $W x^{a}$ could promote RS 2 and RS3 content.

In order to further confirm the above results, a sub-population with total of 16 lines, which only varied in $W x$ locus under the background of CG133R, were selected from 99 lines, of which 6 lines belonged to type $\mathrm{J}$ and 10 lines belonged to type $\mathrm{C}$. Statistical differences were detected in RSm, RSc, RSr, RSa and RSb under different $W x$ alleles by ANOVA, respectively. The result of $t$ test indicated that the average of RS content of type $\mathrm{J}$ significantly differed from type $\mathrm{C}$. The mean values of RSm, RSc, RSr, RSa and RSb of type $\mathrm{C}$ increased by $4.89 \%, 1.00 \%, 1.45 \%, 3.88 \%$ and $1.45 \%$, separately, compared with that of type $\mathrm{J}$ at 0.01 level (Fig. $2 \mathrm{c}$ and d). It could be further confirmed that $W x$ had a great influence on rice RS content that could be elevated by $W x^{a}$.

\section{Effects of SSII-3 on RS in the RIL}

Ninety-nine lines were divided into two groups by SSII-3 alleles: type I and type II, and their RS content distinctly differed from each other in the three sample types ( $P<0.01)$ (Fig. 3a). RSm of type I was 17.83\%, and that of type II only $5.57 \%$. However, the RSc and RSr of type I were lower than that of type II. It showed that SSII-3 alleles polymorphism has an important impact on rice RS, but the specific role need further confirmation. Moreover, the difference of RSa was notable under different SSII-3 alleles by ANOVA $(P<0.01)$ (Fig. 3b). The RSa of type I was $17.27 \%$, which also differed from type II, with a mean of $4.74 \%$. In summary, SSII-3 played an important role in controlling rice RS, except for RS3.

Furthermore, under the CG133R genetic background, 18 lines that only had polymorphisms in SSII-3 locus were selected from RIL, of which 6 lines belonged to type I and 12 lines belonged to type II. Significant differences were detected in RSm or RSa under different SSII-3 alleles by $t$ test. RSm of type I (with a mean of $21.33 \%$ ) was obviously higher than that of type II (with a mean of $5.56 \%$ ). RSa of Type I 


\section{Combined effects of Wx and SSII-3 alleles on RS}

A total of 24 lines, which only separated in Wx and SSII-3 loci under the background of CG133R in the 99 lines, were isolated to analyze their combined effects on RS (Additional file 2: Table S3). Results demonstrated that the interacting effect of $W x$ and SSII-3 alleles for RSc was significant at 0.05 level, and for RSm, RSr and RSa were significant at 0.01 level (Additional file 2: Table S4). The combined effects of $W x$ and SSII-3 alleles on rice RS must be further studied.

There were four combinations among the test rice lines: $\mathrm{J}-\mathrm{I}, \mathrm{J}-\mathrm{II}, \mathrm{C}-\mathrm{I}$ and $\mathrm{C}-\mathrm{II}$, which came from the combination of Wx and SSII-3 alleles (Fig. 4). RSc of the combination C-I (0.89\%) and C-II (1.04\%) was significantly higher than that of $\mathrm{J}-\mathrm{I}(0.061 \%)$ and $\mathrm{J}-\mathrm{II}(0.037 \%)$. Besides that, the significant differences on RSc between homozygotes of $W x^{a}$ and $w x$ were found under the same background of SSII-3 alleles. However, the differences on RSc between homozygotes of different SSII-3 alleles were not significant under the same background of $W x$. And the same result also showed in RSr. These results indicated that $W x$ had an important influence on RSc and RSr under the same background of SSII-3 alleles. Furthermore, RSm of $\mathrm{J}-\mathrm{I}(7.79 \%)$ was similar to that of C-II (5.59\%), which was significantly higher than that of $\mathrm{J}-\mathrm{II}$ (0.67\%), but distinctly lower than C-I (21.33\%) at 0.01 level, and RSa with same effect. These results revealed that different combinations of $W x$ and SSII-3 alleles played vital role in determining RSm and RSa.

\section{Correlation Analysis Between RS And Physicochemical Properties In RIL}

In this study, the physicochemical properties of the RIL and two parents were tested (Additional file 2: Table S5). The correlation analyses between physicochemical properties and RSm, RSc and RSr were carried out. The AAC, all DSC thermal parameters, PKV, PaT and CSV showed a positive correlation with the RSm (Table 2). The AAC and all RVA profile parameters, except PKV, BDV and PaT, were significantly positively correlated with RSc, while all DSC thermal parameters showed significantly negative correlation with RSc $(P<0.05)$, and the same result also showed in RSr. Besides that, all the correlation coefficients of RSr were lower than that of RSc, except for PKV. These data suggested that RS was closely relative with many indexes of physicochemical properties and the correlation between RS and most physicochemical properties were different in raw or gelatinized rice. 
Table 2

Correlations between RS and Physicochemical Properties in RIL

\begin{tabular}{|c|c|c|c|}
\hline & RSm & RSc & $\mathrm{RSr}$ \\
\hline AAC & $0.43^{\star *}$ & $0.849 * \star$ & $0.828 * *$ \\
\hline $\mathrm{GC}$ & 0.096 & $-0.472^{\star \star}$ & $-0.432^{* *}$ \\
\hline T0 & $0.244^{\star \star}$ & $-0.362^{\star \star}$ & $-0.351 * \star$ \\
\hline Tp & $0.56^{* *}$ & $-0.53^{\star \star}$ & $-0.486 \star \star$ \\
\hline Tc & $0.481^{\star \star}$ & $-0.574^{\star \star}$ & $-0.542^{\star \star}$ \\
\hline$\Delta \mathrm{H}$ & $0.236^{\star}$ & $-0.695^{\star \star}$ & -0.667 ** \\
\hline $\mathrm{R} \%$ & $0.531^{\star *}$ & $-0.219 *$ & $-0.204^{\star}$ \\
\hline PKV & $0.288^{\star *}$ & 0.084 & 0.092 \\
\hline HPV & 0.095 & $0.641^{\star *}$ & $0.607 * \star$ \\
\hline CPV & 0.18 & $0.802^{\star \star}$ & $0.781^{\star *}$ \\
\hline PeT & 0.194 & $0.724^{\star \star}$ & $0.69 * *$ \\
\hline РаT & $0.582^{\star \star}$ & $-0.408^{\star *}$ & $-0.388 * \star$ \\
\hline BDV & 0.106 & $-0.672^{\star \star}$ & 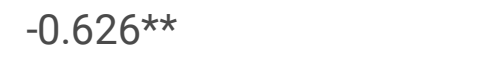 \\
\hline SBV & 0.094 & $0.857 \star \star *$ & $0.83^{\star *}$ \\
\hline CSV & $0.225^{\star}$ & $0.815^{\star \star}$ & $0.807 * *$ \\
\hline \multicolumn{4}{|c|}{$\begin{array}{l}\text { Note: RSm, resistant starch content in milled sample; RSc, resistant starch content in cooked rice; RSr, } \\
\text { resistant starch content in retrograded; AAC, apparent amylose content; GC, gel consistency; T0, } \\
\text { gelatinization start temperature; Tp, gelatinization peak temperature; Tc, gelatinization end } \\
\text { temperature; } \Delta \mathrm{H} \text {, Enthalpy change; R\%, percent of retrogradation; PKV, peak viscosity; HPV, hot paste } \\
\text { viscosity; CPV, cool paste viscosity; PeT, peak time; PaT, pasting temperature; BDV, breakdown value; } \\
\text { SBV, consistence value; SCV, setback value.., ** correlations are significant at } p<0.05, p<0.01 \text {, } \\
\text { respectively. }\end{array}$} \\
\hline
\end{tabular}

\section{Discussion}

RS2 and RS3 are two main subtypes of RS, which are resistant to enzymatic hydrolysis. RS2 is consists of native starch granules. It is reported that RS2 has a unique crystalline structure with B-type X-ray diffraction pattern, which is resistant to the enzymatic digestions (Topping and Clifton, 2001). However, its resistance to enzymatic treatment will greatly decrease after hydrothermal processing (Wang and Copeland, 2013). Chung et al. (2006) found that it was more susceptible to hydrolysis for RS2 after heat process or cooking, and most of RS2 in the native waxy starch will be transformed into enzymatic starch after cooking. In this study, the average of RS content in raw starch samples was $10.61 \%$, but its RS content dramatically decreased after cooking (Table 1). We deduced that the subtype of RS in these 
materials was mainly RS2 according to the characteristics of RS2. RS3 is retrograded starch, mainly coming from the gelatinized starch after cooling (Sharma et al. 2008), thermally very stable and hardly digested. RS content in these plant materials increased by $0.33 \%$ after storage at $4{ }^{\circ} \mathrm{C}$ for 7 days, but the difference was no significant between RSc (with a mean of $0.73 \%$ ) and $\mathrm{RSr}$ (with a mean of $1.06 \%$ ) at 0.05 level (Table 1, Additional file 1: Figure S1). This result revealed that the RS3 in these materials was low. Briefly, the subtype of RS was mainly RS2 in this RILs, while RS3 was less.

Wx is the major gene controlling the amylose synthesis of rice (Tian et al. 2009). Previous studies indicated that Wx could influence the digestibility of starch and it was the major gene controlling Glycemic Index (GI) (Fitzgerald et al. 2011; Raben et al. 1994). Kong et al. (2015) made association test with 14 rice accessions to survey relationship between genetic and functional properties of rice starch and found that Wx could control the amount of RS. In this work, the results of genetic analysis demonstrated that Wx had a great effect on RS content and the Wx from CG133R $\left(W x^{a}\right)$ could improve the RS content in rice (Fig. 2). Wxa is one of the vital alleles of Wx, which produces a large amount of amylose (Itoh et al. 2003). Raigond et al. (2015) found that high amylose could contribute to RS content. Bao et al. (2017) utilized the genome-wide association study to select the candidate genes affecting RS and identified that $\mathrm{Wx}$ was one of the candidate genes, as well as the mean RS content of indica rice was higher than that of the japonica rice. Furthermore, Zhou et al. (2016) revealed that RS production was dependent on the high expression of $\mathrm{Wx}^{\mathrm{a}}$ allele, which is consistence with our conclusion. It can be inferred that Wx could affect RS through controlling amylose content.

SSII-3 plays a vital role in the amylopectin synthesis pathway, and it can control the degree of crystallinity and the amount of short A chains of amylopectin (Kong et al. 2015). As reported in previous articles, SSII3 was found to have an effect on RS content (Bao et al. 2017), and the down-regulation of SSII-3 could enhance RS content in barley (Bird et al. 2004) and wheat (Yamamori et al. 2006). SSII-3 is present in a large complex including ADP-glucose pyrophosphorylase (AGPase), pyruvate orthophosphate dikinase (PPDK), SSIlla, SBElla and SBEllb, which regulate the partitioning of ADP-glucose (ADPGIc) into lipid or starch and further control RS5 (Zhou et al. 2016). However, Itoh et al. (2017) employed 7 rice lines to survey the pleiotropic effects of biosynthetic enzymes and indicated that SSII-3 could change the distribution of amylopectin chain but may not affect RS level. Our study showed that SSII-3 had an influence on rice RS except for RS3 in RIL (Fig. 3a and b), but only affected RS content in raw starch and RS2 in 18 lines (Fig. 3c and d). In 18 lines, the RSm and RSa of lines with SSII-3 allele from Javanica 22 (with a mean of $21.33 \%$ and $20.44 \%$, respectively) were significantly higher than that from CG133R ( $5.56 \%$ and $4.52 \%$, respectively) (Fig. $3 \mathrm{c}$ and d), which may be caused by the structure of amylopectin and need further study. Given that Wx and SSII-3 are closely located on chromosome 6, therefore, the interactions of the two genes on RS were analyzed. Apart from RS3, the combined effects of Wx and SSII3 greatly affected RSm, RSc, RSr and RS2 (Additional file 2: Table S4). The RSc and RSr of C-I (with a mean of $0.89 \%$ and $1.283 \%$, respectively) and C-II (1.04\% and $1.49 \%)$ were higher than that of $\mathrm{J}-\mathrm{I}(0.06 \%$ and $0.15 \%)$ and $\mathrm{J}-\mathrm{II}(0.04 \%$ and $0.05 \%)$. Besides that, the statistical differences on RSc were identified between $\mathrm{W} \mathrm{x}^{\mathrm{a}}$ and $\mathrm{wx}$ alleles under the same background of SSII-3 alleles, but the difference between the 
different SSII-3 alleles was not significant under the same Wx allele (Fig. 4b and c). This result indicated that Wx had a great impact on RS from cooked rice and retrograded rice under the same background of SSII-3 alleles.

In addition to affecting RS content, the amylose content and amylopectin fine structure also determines the physicochemical properties of rice starch. Therefore, it is necessary to analyze the relationship between RS content and physicochemical properties of rice. Shu et al. (2014) and Zhang et al. (2007) revealed that AAC was positively correlated with RS. Furthermore, Ding et al. (2019) found that the effect of amylose content on RS in raw rice was not as great as that on RS in cooked rice, which is consistent with our result and the correlation coefficient between AAC and RSm was smaller than that between AAC and $R S c\left(R^{2}=0.43,0.849\right.$, respectively, $P<0.01$; Table 2$)$. The correlation of RVA parameters and RSm were different from that and RSc. The former had a positive correlation at PKV, PaT and CSV, but the later with significantly positive correlation for most of RVA profile parameters. The results were similar to the previous result of correlation analysis reported by Ding et al. (2019). Furthermore, Chen et al. (2017) analyzed the correlation between RS and physiochemical properties of cooked rice and results showed that RS was negatively correlated with PKV and BDV. Nevertheless, Kong et al. (2015) indicated that RS had no correlation with PKV and BDV. Nakamura et al. (2015) found that RS was positively correlation with PKV and BDV. In our study, a negatively correlation between RS and BDV was detected $\left(R^{2}=-0.672, P\right.$ $<0.01$; Table 2), but the relationship between RS and PKV was not observed. Moreover, it was reported that lower GT correlated with higher starch digestibility in vitro although their relationship was not statistically significant (Dhital et al. 2015). However, Yang et al. (2006) found that mutant RS111 with high RS content showed notably lower To, Tp, Tc and $\Delta \mathrm{H}$ values, compared with those of wild type and common rice. In present study, the gelatinization temperatures such as To, $\mathrm{Tp}, \mathrm{Tc}, \mathrm{R} \%$ and $\Delta \mathrm{H}$ were negatively correlated with RSc, but positively correlated with RSm $(P<0.01)$.

\section{Conclusions}

Previous studies mainly focused on investigating RS in raw rice or cooked rice separately, which may receive different results. In present study, the RS contents of 99 lines were measured for them in different processing status. RS contents of 99 lines were sharply decreased after cooking, but no distinct variation was observed in RS content after cooling (Additional file 1: Figure S1). Thus, we deduced that the subtype of RS in present study was mostly RS2 and less RS3 according to the characteristics of RS2 and RS3. The results of genetic analysis demonstrated that Wx had a great effect on RS content and W $x^{a}$ could elevate RS content in different processing rice(Fig. 2). The different SSII-3 alleles resulted in the variation of RS in raw rice and RS2 (Fig. 3). Furthermore, the combined effects of Wx and SSII-3 was responsible for the variation of RS content in three sample types and RS2. For cooked and retrograded rice, effects of Wx alleles on RS were significant different under the same background of SSII-3 alleles (Fig. 4). RS was closely relative with many indexes of physicochemical properties (Table 2). The study shows that the RS content in rice will change by different processing ways, moreover, Wx and SSII-3 play an vital role in controlling rice RS, which may provide useful information for molecular breeding of rice RS. 


\section{Abbreviations}

RS: resistant starch; SCFA: short chain fatty acids; ECQs: eating and cooking qualities; AAC: apparent amylose content; GC: gel consistency; GT: gelatinization temperature; RVA: rapid visco analyzer; RIL: recombinant inbred line; DSC: differential scanning calorimetry; R\%: percentage of retrogradation; PKV: peak viscosity; HPV: hot paste viscosity; CPV: cool paste viscosity; BDV: breakdown viscosity; SBV: setback viscosity; CSV: consistence viscosity; PaT: pasting temperature; PeT: pasting time; GI: glycemic index.

\section{Declarations}

\section{Ethics Approval and Consent to Participate}

Not applicable

\section{Consent for Publication}

Not applicable

\section{Availability of Data and Materials}

All data generated or analysed during this study are available within in this article or its supplementary files.

\section{Competing Interests}

The authors declare that they have no competing interests.

\section{Funding}

This work was supported by the Key Research \& Development program of Sichuan Province, China (Grant No. 2019YFN0019), and the Key Program of Education Bureau of Sichuan Province, China (Grant No. 17ZA0412).

\section{Authors' Contributions}

$\mathrm{HY}$ and $\mathrm{LX}$ investigated genetic studies. HY, LX and $\mathrm{OZ}$ carried out the measure of resistant starch and physicochemical properties. XX designed the overall project. HY analyzed the data and wrote the manuscript. All authors read and approved the final manuscript.

\section{Acknowledgements}

Not applicable

\section{Authors details}


${ }^{1}$ Lab of Plant Molecular Genetics and Breeding, Southwest University of Science and Technology, 59 Qinglong Road, Mianyang 621010, China.

\section{References}

1. Ahmed R, Pierre B, Behjat KH, Suzhi L, Mark C, Oscar L, Anthony RB, Steve MS, Colin C, Stephen AJ, Zhong YL, Matthew M (2015) A genetic strategy generating wheat with very high amylose content. Plant Biotechnol J 13:1276-1286

2. Asp NG (1992) Preface resistant starch - Proceedings from the second plenary meeting of EURESTA European FLAIR Concerted Action no. 11 on physiological implications of the consumption of resistant starch in man. Eur J Clin Nutr 46:S1-148

3. Bao J, Zhou X, Xu F, He Q, Park YJ (2017) Genome-wide association study of the resistant starch content in rice grains. Starch - Stärke 69:1600343

4. Bird AR, Flory C, Davies DA, Usher S, Topping DL (2004) A novel barley cultivar (Himalaya 292) with a specific gene mutation in starch synthase lla raises large bowel starch and short-chain fatty acids in rats. J Nutr 134:831-835

5. Cai XL, Liu QQ, Tang SZ, Gu MH, Wang ZY (2002) Development of a molecular marker for screening the rice cultivars with intermediate amylose content in Oryza sativa indica. Acta photophysiologica sinica 28:137-144

6. Chen MH, Bergman CJ, McClung AM, Everette JD, Tabien RE (2017) Resistant starch: variation among high amylose rice varieties and its relationship with apparent amylose content, pasting properties and cooking methods. Food Chem 234:180-190

7. Chung HJ, Lim HS, Lim ST (2006) Effect of partial gelatinization and retrogradation on the enzymatic digestion of waxy rice starch. J Cereal Sci 43:353-359

8. Dhital S, Dabit L, Zhang B, Flanagan B, Shrestha AK (2015) In vitro digestibility and physicochemical properties of milled rice. Food Chem 172:757-765

9. Ding Y, Huang J, Zhang N, Rasmussen SK, Wu D, Shu X (2019) Physiochemical properties of rice with contrasting resistant starch content. J Cereal Sci 89:102815

10. Eggum BO, Juliano BO, Perez CM, Acedo EF (1993) The resistant starch, undigestible energy and undigestible protein contents of raw and cooked milled rice. J Cereal Sci 18: 159-170

11. Fitzgerald MA, Rahman S, Resurreccion AP, Concepcion J, Daygon VD, Dipti SS, Kabir KA, Klingner B, Morell MK, Bird AR (2011) Identification of a major genetic determinant of glycaemic index in rice. Rice 4:66-74

12. Frei $M$, Siddhuraju P, Becker $K$ (2003) Studies on the in vitro starch digestibility and the glycemic index of six different indigenous rice cultivars from the Philippines. Food Chem 83:395-402

13. Hazard B, Zhang X, Naemeh M, Dubcovsky J (2014) Registration of durum wheat germplasm lines with combined mutations in SBElla and SBEIlb genes conferring increased amylose and resistant starch. J Plant Regist 8:334-338 
14. Hernández O, Emaldi U, Tovar J (2008) In vitro digestibility of edible films from various starch sources. Carbohyd Polym 71:648-655

15. Hu P, Zhao H, Duan Z, Lin ZL, Wu D (2004) Starch digestibility and the estimated glycemic score of different types of rice differing in amylose contents. J Cereal Sci 40:231-237

16. Itoh K, Ozaki H, Okada K, Hori H, Takeda Y, Mitsui T (2003) Introduction of Wx transgene into rice wx mutants leads to both high- and low-amylose rice. Plant Cell Physiol 44: 473-480

17. Itoh Y, Crofts N, Abe M, Hosaka Y, Fujita N (2017) Characterization of the endosperm starch and the pleiotropic effects of biosynthetic enzymes on their properties in novel mutant rice lines with high resistant starch and amylose content. Plant Sci 258:52-60

18. Kim JH, Tanhehco EJ, Ng PKW (2006) Effect of extrusion conditions on resistant starch formation from pastry wheat flour. Food Chem 99:718-723

19. Kong X, Chen Y, Zhu P, Sui Z, Corke H, Bao J (2015) On the relationships among genetic, structural and functional properties of rice starch. J Agr Food Chem 63:6241-6248

20. Murray MG, Thompson WF (1980) Rapid isolation of high molecular weight plant DNA. Nucleic Acids Res 8:4321-4326

21. Nakamura S, Katsura J, Kato K, Ohtsubo Ki (2015) Development of formulae for estimating amylose content and resistant starch content based on the pasting properties measured by RVA of Japonica polished rice and starch. Biosci Biotech Bioch 80:329-340

22. Raben A, Tagliabue A, Christensen NJ, Madsen J, Holst JJ, Astrup A (1994) Resistant starch: the effect on postprandial glycemia, hormonal response, and satiety. Am J Clin Nutr 60: 544-551

23. Raigond P, Ezekiel R, Raigond B (2015) Resistant starch in food: a review. J Sci Food Agr 95:19681978

24. Robertson JA, Monredon FDD, Dysseler P, Guillon F, Amado R, Thibault JF (2000) Hydration properties of dietary fibre and resistant starch: a european collaborative study. LWT - Food Sci Technol 33:72-79

25. Sharma A, Yadav BS, Ritika (2008) Resistant starch: physiological roles and food applications. Food Rev Int 24:193-234

26. Shu X, Sun J, Wu D (2014) Effects of grain development on formation of resistant starch in rice. Food Chem 164:89-97

27. Tian Z, Qian Q, Liu Q, Yan M, Liu X, Yan C, Liu GF, Gao ZY, Tang SZ, Zeng DL, Wang YH, Yu JM, Gu $\mathrm{MH}$, Li JY (2009) Allelic diversities in rice starch biosynthesis lead to a diverse array of rice eating and cooking qualities. Proc Natl Acad Sci U S A 106:21760-21765

28. Tian Z, Yan C, Qian Q, Yan S, Xie H, Wang F, Xu JF, Liu G, Wang YH, Liu QQ, Tang S, Li JY, Gu MH (2010) Development of gene-tagged molecular markers for starch synthesis-related genes in rice. Chinese Sci Bull 33:3768-3777

29. Topping DL, Bajka BH, Bird AR, Clarke JM, Cobiac L, Conlon MA, Morell MK, Toden S (2008) Resistant starches as a vehicle for delivering health benefits to the human large bowel. Microb Ecol Health Dis 
20:103-108

30. Topping DL, Clifton PM (2001) Short-chain fatty acids and human colonic function: roles of resistant starch and nonstarch polysaccharides. Physiol Rev 81:1031-1064

31. Topping DL, Morell MK, King RA, Li Z, Bird AR, Noakes M (2003) Resistant starch and health Himalaya 292, a novel barley cultivar to deliver benefits to consumers. Starch - Stärke 55:539-545

32. Wang S, Copeland L (2013) Molecular disassembly of starch granules during gelatinization and its effect on starch digestibility: a review. Food Funct 4:1564-1580

33. Xia J, Zhu D, Wang R, Cui Y, Yan Y (2018) Crop resistant starch and genetic improvement: a review of recent advances. Theor Appl Genet 131:2495-2511

34. Yamamori M, Kato M, Yui M, Kawasaki M (2006) Resistant starch and starch pasting properties of a starch synthase lla-deficient wheat with apparent high amylose. Aust J Agr Res 57: 531-535

35. Yan CJ, Fang, YW, Li M, Peng JC, Liu QQ, Tang SZ, Gu MH (2010) Genetic effect of PUL allelic variation on rice cooking and eating qualities. Acta Agronomica Sinica 36:728-735

36. Yang CZ, Shu XL, Zhang LL, Wang XY, Zhao HJ, Ma CX, Wu DX (2006) Starch properties of mutant rice high in resistant starch. J Agr Food Chem 54:523-528

37. Yang R, Sun CL, Bai JJ, Lou ZX, Shi B, Zhang JM, Yan WG, Piao ZZ (2012) A putative gene sbe3-rs for resistant starch mutated from SBE3 for starch branching enzyme in rice (Oryza sativa). Plos One 7:e43026

38. Zhang W, Bi J, Yan X, Wang H, Zhu C, Wang J, Wan J (2007) In vitro measurement of resistant starch of cooked milled rice and physico-chemical characteristics affecting its formation. Food Chem 105:462-468

39. Zhou H, Wang L, Liu G, Meng X, Jing Y, Shu X, Kong X, Sun J, Yu H, Smith SM, Wu D, Li J (2016) Critical roles of soluble starch synthase SSIlla and granule-bound starch synthase Waxy in synthesizing resistant starch in rice. Proc Natl Acad Sci U S A 113:12844-12849

\section{Figures}




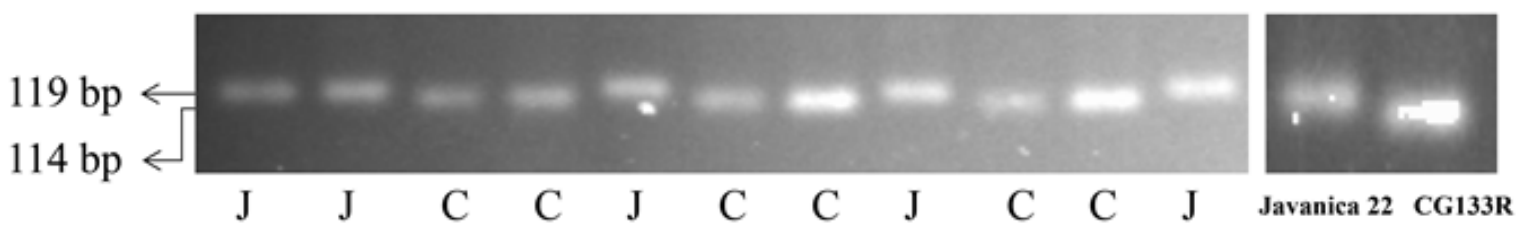

AGPlar M1
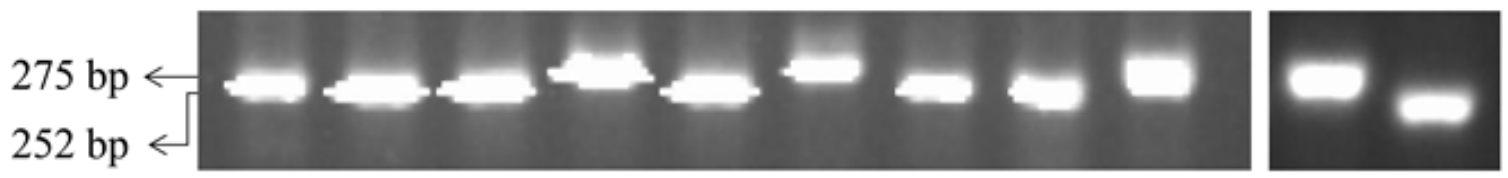

Wx M1

$\begin{array}{lllllllllllll}\mathrm{C} & \mathrm{C} & \mathrm{C} & \mathrm{J} & \mathrm{C} & \mathrm{J} & \mathrm{C} & \mathrm{C} & \mathrm{J} & \text { Javanica } 22 & \text { CG133R }\end{array}$
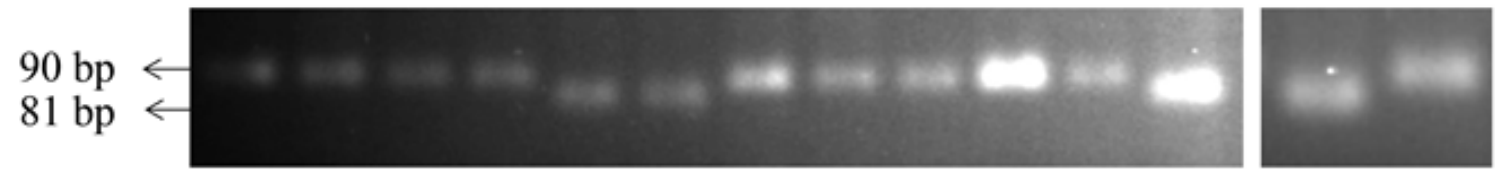

SSII-3 M1

$\begin{array}{llllllllllllllll}\text { II } & \text { II } & \text { II } & \text { II } & \text { I } & \text { I } & \text { II } & \text { II } & \text { II } & \text { II } & \text { II } & \text { I } & \text { Javanica } 22 & \text { CG 133R }\end{array}$

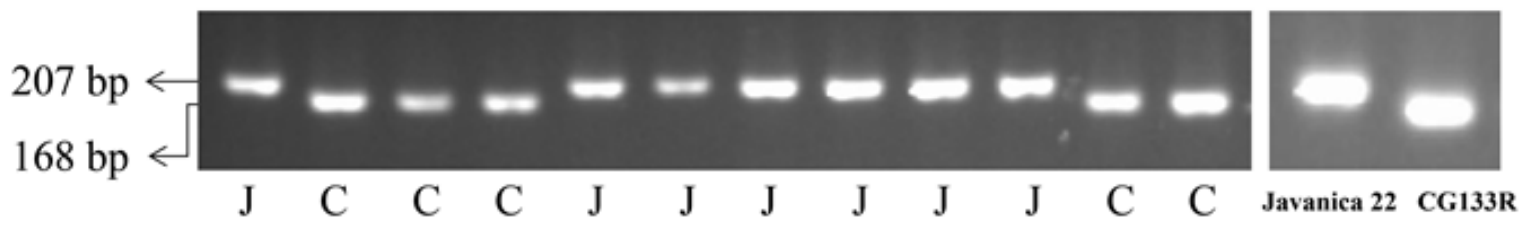

AGPsma 1

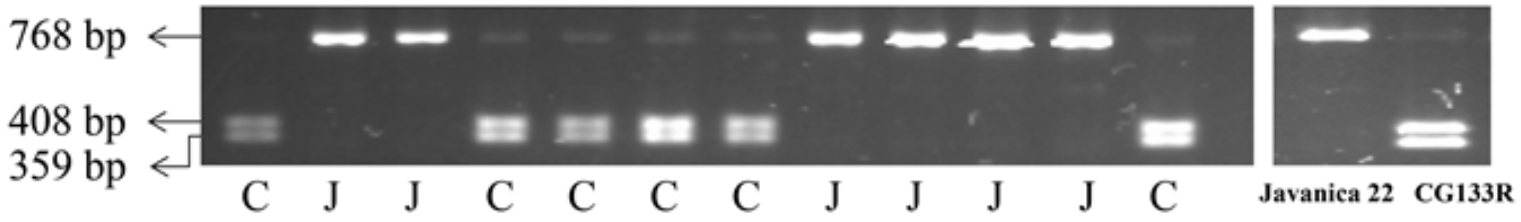

SSIII-1 t 1

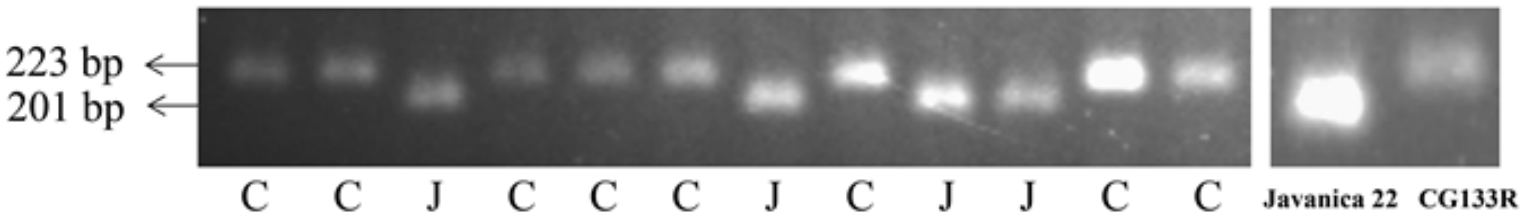

$S S I V-2 \mathrm{M} 2$

\section{Figure 1}

Polymorphism gene loci of starch synthesis-related genes between two parents and partial tested materials. $J$ and $C$ indicate genotype of Janavica 22 and CG133R, respectively; $\otimes$ and $\otimes$ indicate genotype of Janavica 22 and CG133R, respectively. 

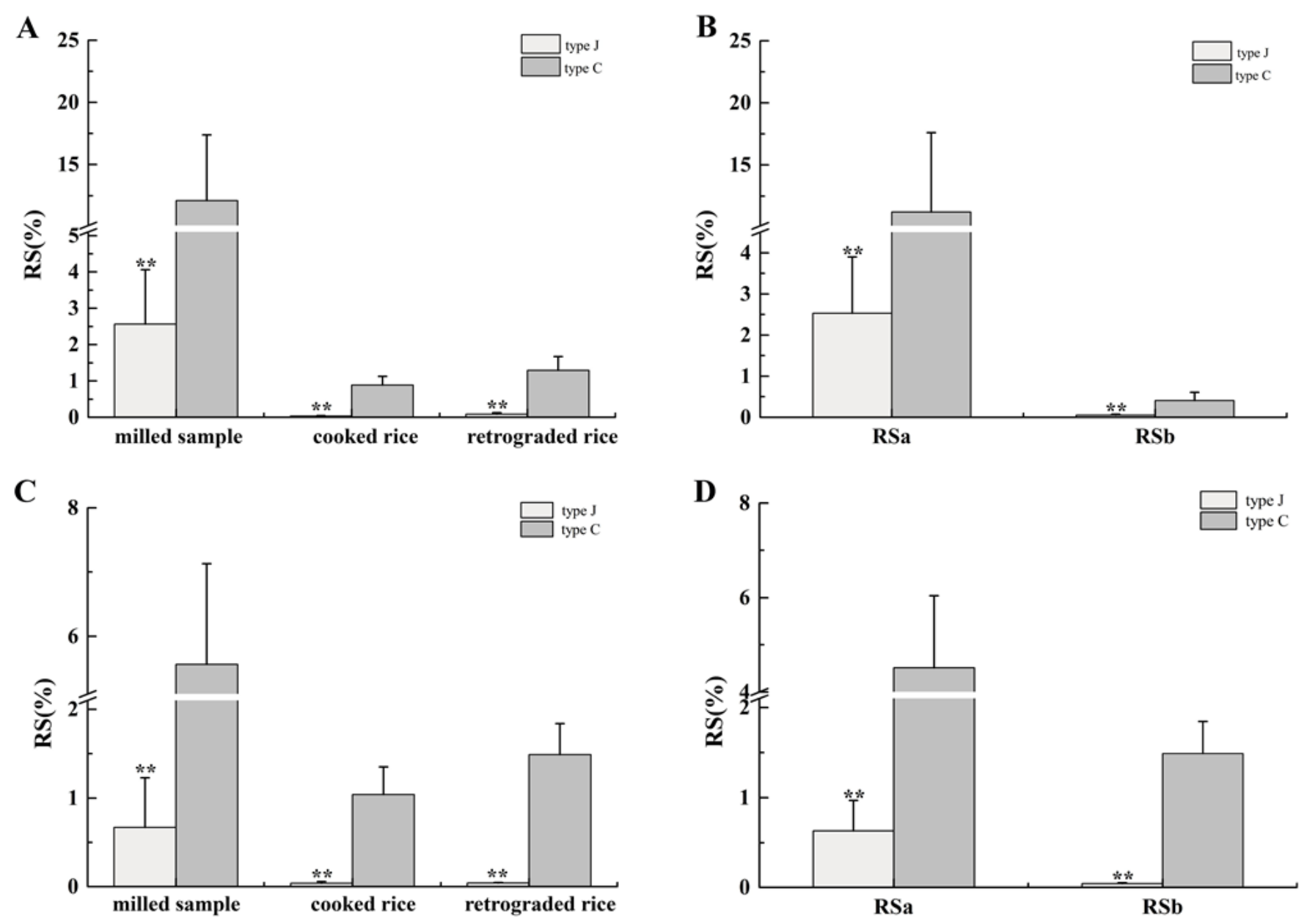

\section{Figure 2}

Comparison of RS among different Wx genotypes in RIL. Type J, C indicate homozygote for Janavica 22 and CG133R in Wx allele, respectively; RSa= RSm-RSc; RSb= RSr-RSc. (a) RS content in milled sample, cooked rice and retrograded rice at different Wx alleles in the RIL. (b) RSa and RSb content at different Wx alleles in the RIL. (c) RS content in milled sample, cooked rice and retrograded rice at different Wx alleles in the 16 lines. (d) RSa and RSb content at different Wx alleles in the 16 lines. Each column represents the mean \pm standard deviation, $* *$ indicates $P<0.01$. 

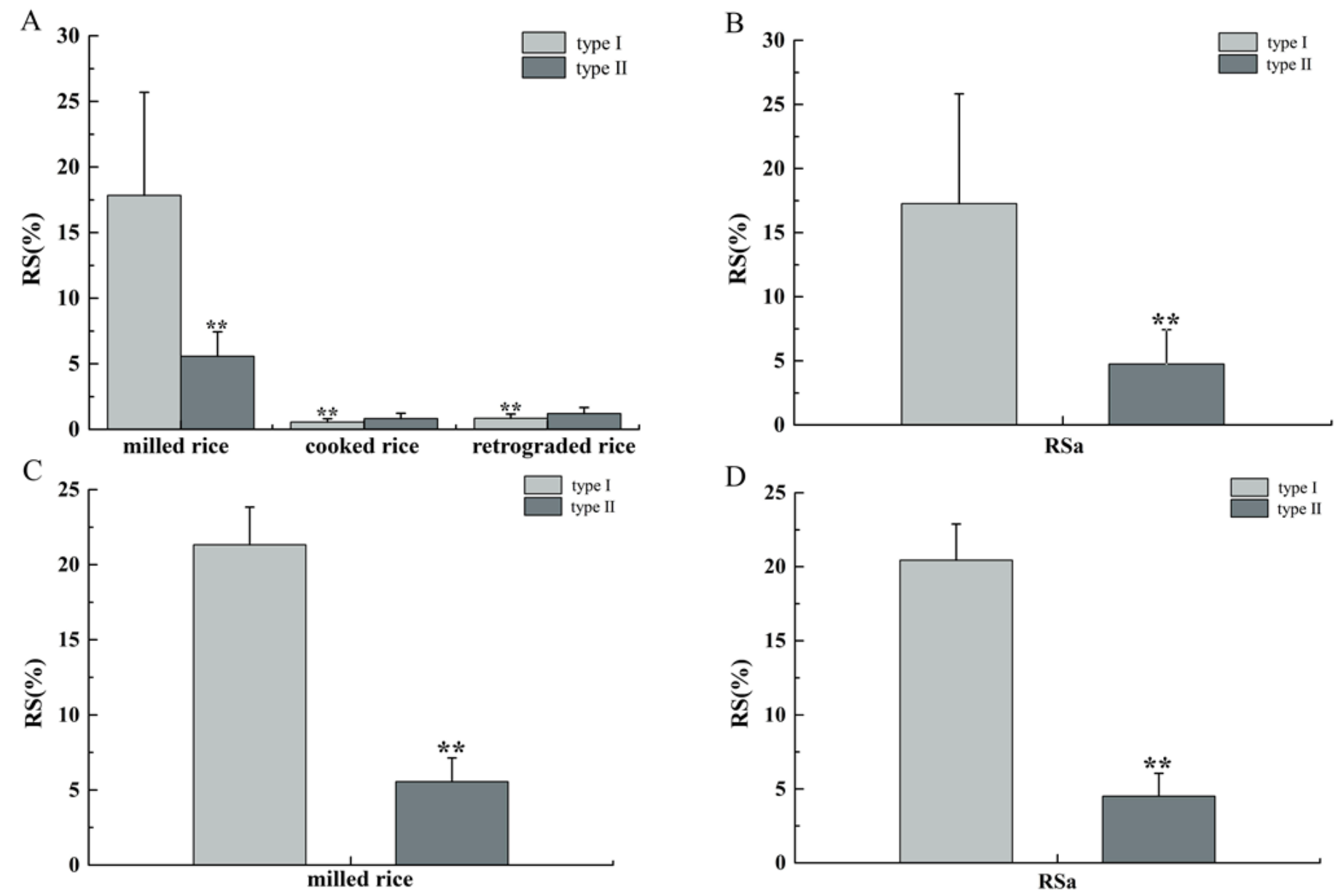

\section{Figure 3}

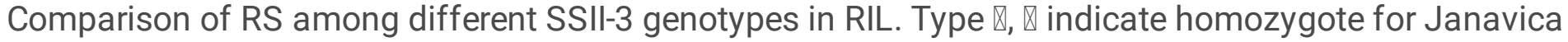
22 and CG133R in SSII-3 locus, respectively; RSa= RSm-RSc. (a) RS content in milled sample, cooked rice and retrograded rice at different SSII-3 alleles in the RIL. (b) RSa at different SSII-3 alleles in the RIL. (c) RS content in milled sample at different SSII-3 alleles in the 18 lines. (d) RSa at different SSII-3 alleles in the 18 lines. Each column represents the mean \pm standard deviation, $* *$ indicates $P<0.01$. 

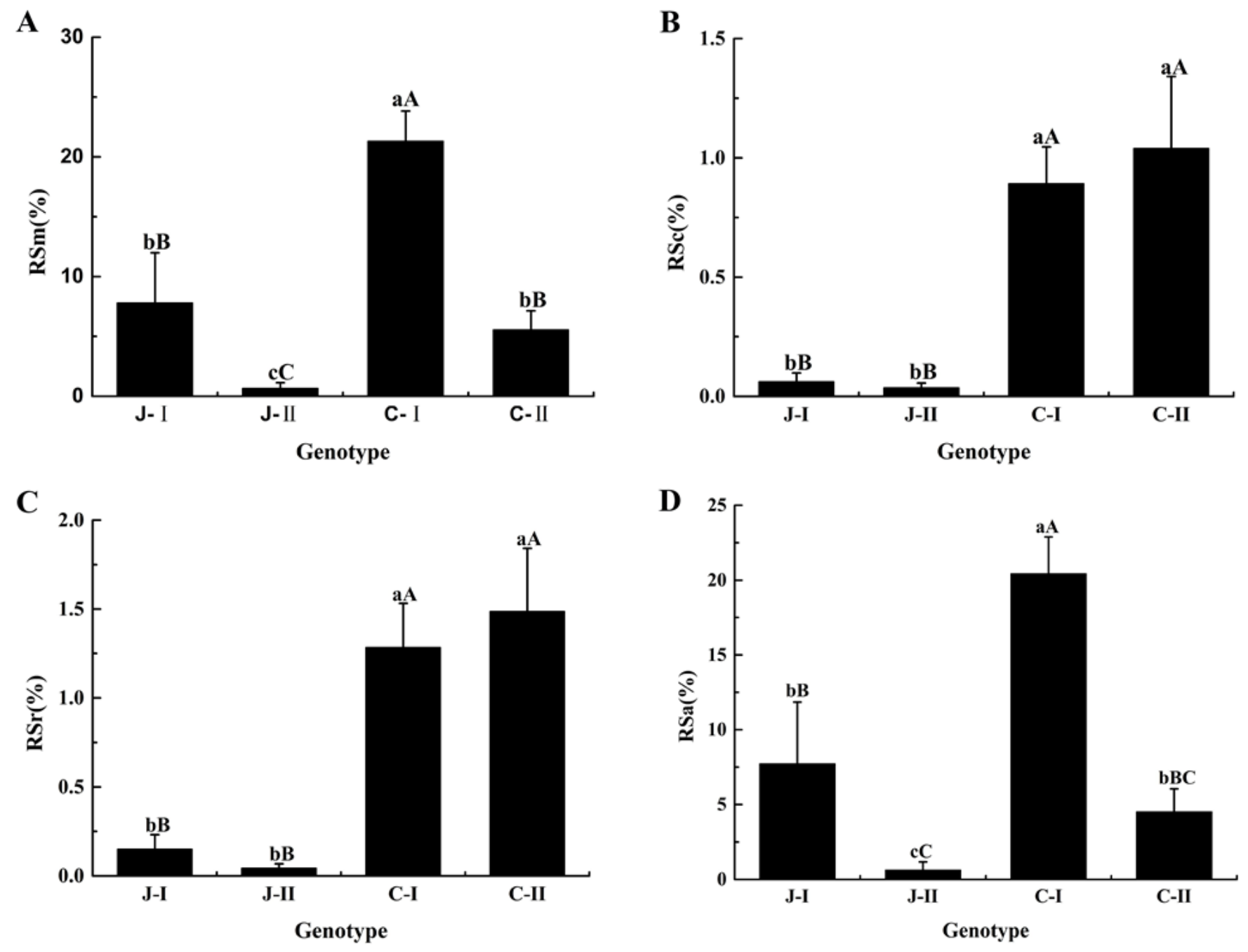

\section{Figure 4}

Fig. 4 Comparison of RS for different combinations of Wx $\times$ SSII-3. Type J, C indicate homozygote for Janavica 22 and CG133R in Wx allele, respectively; Type I, II indicate homozygote for Janavica 22 and CG133R in SSII-3 locus, respectively; RSm, resistant starch content in milled sample; RSc, resistant starch content in cooked rice; RSr, resistant starch content in retrograded rice; RSa= RSm-RSc. (a) RS content in milled sample at different combinations of Wx $\times$ SSII-3. (b) RS content in cooked rice at different combinations of $\mathrm{Wx} \times \mathrm{SSII}-3$. (c) RS content in retrogaded rice at different combinations of $\mathrm{Wx} \times \mathrm{SSII}-3$. (d) RSa at different combinations of Wx $\times$ SSII-3. Lowercase and capital letters above column denote significant at 0.05 and 0.01 levels, respectively.

\section{Supplementary Files}

This is a list of supplementary files associated with this preprint. Click to download.

- supplementarymaterials.doc 
Page 20/20 